\title{
BUBR1 Insufficiency Is Correlated with eNOS Reduction Experimentally In Vitro and In Vivo, and in Gastric Cancer Tissue
}

\author{
EISUKE KAWAKUBO ${ }^{1}$, TAKUYA MATSUMOTO ${ }^{1,2}$, KEIJI YOSHIYA ${ }^{1}$, SHO YAMASHITA ${ }^{1}$, TOMOKO JOGO $^{1}$, \\ HIROSHI SAEKI ${ }^{1}$, EIJI OKI ${ }^{1}$, TADASHI FURUYAMA ${ }^{1}$, YOSHINAO ODA ${ }^{3}$ and YOSHIHIKO MAEHARA ${ }^{1}$ \\ Departments of ${ }^{1}$ Surgery and Science, and ${ }^{3}$ Anatomic Pathological Sciences, \\ Graduate School of Medical Sciences, Kyushu University, Fukuoka, Japan; \\ ${ }^{2}$ Department of Medicine, International University of Health and Welfare, Narita, Japan
}

\begin{abstract}
Background/Aim: Budding uninhibited by benzimidazole-related 1 (BUBR1) and endothelial nitric oxide synthase (eNOS) are related to aging and angiogenesis. This study examined the effect of low BUBRI expression on eNOS expression in vivo, in vitro, and human gastric cancer tissues. Materials and Methods: Human umbilical vein endothelial cells (HUVECS) were passaged to investigate the effect of aging on BUBRI and eNOS expression; expression of eNOS and phospho-eNOS protein was assessed in BUBRI siRNAtransfected HUVECs. Additionally, guanosine 3',5' cyclic monophosphate (cGMP) and eNOS protein levels were measured in BUBR1-insufficient mice (Bubr $\left.{ }^{L /-}\right)$. BUBR1 and eNOS expression levels were also evaluated in human gastric cancer tissues. Results: BUBRI and eNOS, but not p-eNOS, levels were reduced significantly in aged and BUBRI siRNAtransfected HUVECs. Additionally, cGMP production and the eNOS protein level were reduced in Bubr1 ${ }^{L /-}$ mice. Human gastric cancer tissues with low BUBRI expression showed no eNOS expression. Conclusion: A decrease in BUBR1 reduced eNOS bioavailability through a pathway other than eNOS phosphorylation.
\end{abstract}

Both aging and angiogenesis are critical risk factors for the development of cancer and cardiovascular diseases (1).

Correspondence to: Yoshihiko Maehara, Department of Surgery and Science, Graduate School of Medical Sciences, Kyushu University, 3-1-1 Maidashi, Higashiku, Fukuoka 812-8582, Japan. Tel: +81926425466, Fax: +81926425462,e-mail: maehara@ surg2.med.kyushu-u.ac.jp and Takuya Matsumoto, Department of Medicine, International University of Health and Welfare, 4-3 Kouzunomori, Narita 286-8686, Japan. Tel: +81 476207701, Fax: +81476207702, e-mail: tak@iuhw.ac.jp

Key Words: BUBR1, eNOS, aging, angiogenesis.
Aging, i.e. the inevitable time-dependent decline in organ function and tissue viability, advances cancer initiation and progression in many ways $(1,2)$. A variety of age-dependent changes, such as aneuploidy and DNA damage, occur at the cellular level in tissues (3). Aging also has a remarkable effect on endothelial function, leading to an increase in cardiovascular diseases including atherosclerosis, myocardial infarction, and stroke. Notably, endothelial dysfunction is one of the major phenotypic changes in aged arteries $(4,5)$.

Angiogenesis triggered by chemical signals from tumour cells makes tumours and metastasis progress rapidly (6). In contrast, therapeutic angiogenesis using fibroblast growth factor 2 (FGF2) (7), hepatocyte growth factor (HGF) (8), or vascular endothelial growth factor (VEGF) (9) has been widely regarded as an attractive approach for treating ischaemic limbs and for improving endothelial function (10).

Budding uninhibited by benzimidazole-related 1 (BUBR1) is an essential molecule in the spindle assembly checkpoint. The anaphase-promoting complex/cyclosome (APC/C) is inactivated by checkpoint protein complexes containing BUBR1 [mitotic arrest-deficient 3 (MAD3) in yeast], budding uninhibited by benzimidazole 3 (BUB3), MAD2 and cell-division cycle protein 20 (CDC20) until all kinetochores are attached to microtubules in an appropriate manner (11). Previous work demonstrated that BUBR1 overexpression is observed in malignant tumours derived from many organs, such as the stomach and liver (12). Notably, other studies demonstrated that mice expressing only $10 \%$ of normal BUBR1 level develop progressive aneuploidy and many aging phenotypes, such as cataracts, lordokyphosis, and loss of subcutaneous fat (13). In addition, specific early aging-associated vascular phenotypes were observed in these mice, including a reduced number of smooth muscle cells, reduced amount of elasticity, impairment of endothelial-dependent relaxation, and increased production of superoxide anions (5). BUBR1 
insufficiency impairs angiogenesis and results in limb loss in ischaemic hind limbs through suppressing VEGF upregulation (14).

Nitric oxide (NO) is produced from the conversion of L-arginine to L-citrulline by the enzyme endothelial nitric oxide synthase (eNOS) (15). eNOS is responsible for endothelium-derived NO production (16). To function in this role, eNOS must first be activated via phosphorylation (17). The activity of eNOS is regulated by many steps and acts in concert with various mediators (18).

Previous work suggested that in tumour tissues, eNOS induces angiogenesis and recruits perivascular cells, both of which promote tumour growth or metastasis, via NO production (19). In contrast, another study reported that eNOS-derived NO inhibits tumour metastasis (20).

Notably, both the level of expression and activity of eNOS are reduced by aging (21). The loss of NO activity is a central mechanism responsible for dysfunction of the vascular endothelium. An aging-induced increase in the formation of oxygen-derived free radicals appears to be the most likely explanation for the loss of NO (22).

Suppressor of sensor kinase 1 (SSK1) is related to numerous serine-threonine kinases that have high homology with BUBR1. The identification of SSK1 as a cDNA fragment overexpressed following laminar shear stress (23) suggests that BUBR1 could have a relationship with eNOS. Furthermore, the connection between BUBR1 and eNOS may be shared by both aging and angiogenesis. However, the relationship or interaction between BUBR1 and eNOS has not yet been investigated as far as we are aware. Here, we examined the influence of BUBR1 reduction on eNOS expression and activity by performing experiments in vitro, in vivo, and on human gastric cancer tissues.

\section{Materials and Methods}

Cell culture. Human umbilical vein endothelial cells (HUVECs) were obtained from a commercial provider (CC-2519; Lonza, Walkersville, MD, USA). HUVECs were cultured according to the supplier's instructions (CC-2535; Lonza). Cells at passages 4-9 were used for experiments and were plated in triplicate for each experimental condition.

Experimental animals. All animal experiments were conducted in accordance with experimental protocols approved by the University Committee of the Kyushu University Health Science Center (approval no. A24-157-01).

Low-BUBR1-expressing mutant mice (Bubrl ${ }^{\mathrm{L} /-}$ mice) and wildtype (WT) littermates $\left(B u b r I^{+/+}\right)$with a mixed genetic background of C57BL/6J and 129/SvJ were generated in our laboratory, as previously described (14). The thymuses of experimental mice were frozen for use in western blotting and 3',5' cyclic monophosphate (cGMP) measurement. Mice were sacrificed at 7 weeks old for western blotting analysis and at 18-20 weeks old for cGMP measurement.
HUVEC transfection with small interfering RNA (siRNA). BUBR1 siRNA was purchased from Santa Cruz Biotechnology, Inc. (Dallas, TX, USA). As control groups, a group transfected with scrambled siRNA (Invitrogen ${ }^{\mathrm{TM}}$, Carlsbad, CA, USA), and a group without siRNA transfection were included.

Protein extraction and western blot analysis. Total cellular proteins and murine thymus proteins were prepared as previously described (14). Membranes were incubated overnight at $4^{\circ} \mathrm{C}$ with primary: mouse monoclonal antibodies directed against BUBR1 (diluted 1:1000; BD Biosciences, Franklin Lakes, NJ, USA); eNOS (diluted 1:1000; BD Biosciences); or $\beta$-actin (diluted 1:2000; BioVision, Milpitas, CA, USA); with rabbit monoclonal antibodies directed against phospho(p)-eNOS (Ser 1177; diluted 1:1000; Cell Signaling, Danvers, MA, USA); or with rabbit polyclonal antibodies directed against BUBR1 (diluted 1:1000; Littleton, Novus Biologicals, CO, USA); or $\alpha$-tubulin (diluted 1:1000; Abcam, Cambridge, UK).

RNA extraction and real-time polymerase chain reaction (RT-PCR). Total RNA of HUVECs was extracted using ISOGEN reagent (NIPPON GENE, Tokyo, Japan) according to the manufacturer's protocol. Total RNA was reverse transcribed to cDNA using the SuperScript III First-strand SuperMix (Invitrogen) according to the manufacturer's protocol.

For quantitative RT-PCR, $25 \mu \mathrm{l}$ of amplification mixtures (Applied Biosystems StepOnePlus ${ }^{\mathrm{TM}}$ SYBR Green; Thermo Fisher Scientific, Waltham, MA, USA) were prepared containing cDNA (equivalent to 12.5-50.0 ng of reverse-transcribed RNA) and 0.4$1.0 \mu \mathrm{M}$ primers according to the manufacturer's instructions. Reactions were run on an Applied Biosystems StepOnePlus ${ }^{\mathrm{TM}}$. Each sample was analysed in triplicate and normalized to the level of $\beta$ actin mRNA. The PCR protocol was an initial denaturation at $95^{\circ} \mathrm{C}$ for $5 \mathrm{~min}$ followed by 40 cycles of $95^{\circ} \mathrm{C}$ for $10 \mathrm{~s}, 60-65^{\circ} \mathrm{C}$ for $20 \mathrm{~s}$, and $72^{\circ} \mathrm{C}$ for $10 \mathrm{~s}$. Specific primer pairs used for the reactions were previously described $(2,24,25)$.

VEGF treatment. After HUVECs were cultured under each experimental condition, $50 \mathrm{ng} / \mathrm{ml}$ recombinant human VEGF 165 (R\&D systems, Minneapolis, MN, USA) was added to the medium, and the cells were then cultured for $10 \mathrm{~min}$ (26). Cell lysates were then prepared as above, and each protein was measured by western blotting.

cGMP assay. The function of endothelial cells of mice was assessed based on the level of cGMP, which was measured in lysates of the murine thymuses using an EIA kit from Cayman Chemical Inc. (Ann Arbor, MI, USA), as described in the manufacturer's instructions.

Human gastric cancer tissue samples. This study included samples from 106 unselected Japanese patients with primary gastric cancer, all of whom underwent a gastrectomy between 1994 and 2006 at the Department of Surgery and Science, Graduate School of Medical Sciences, Kyushu University Hospital. The patients were 72 men and 34 women, ranging in age from 29 to 86 years (mean=63.6 years). Disclosure of the study information on our homepage was substituted for written informed consent (approval no. 29-396). Thorough histological examination was made using hematoxylin-eosin-stained tissue preparations. No patient treated preoperatively with cytotoxic drugs was included in this study. 


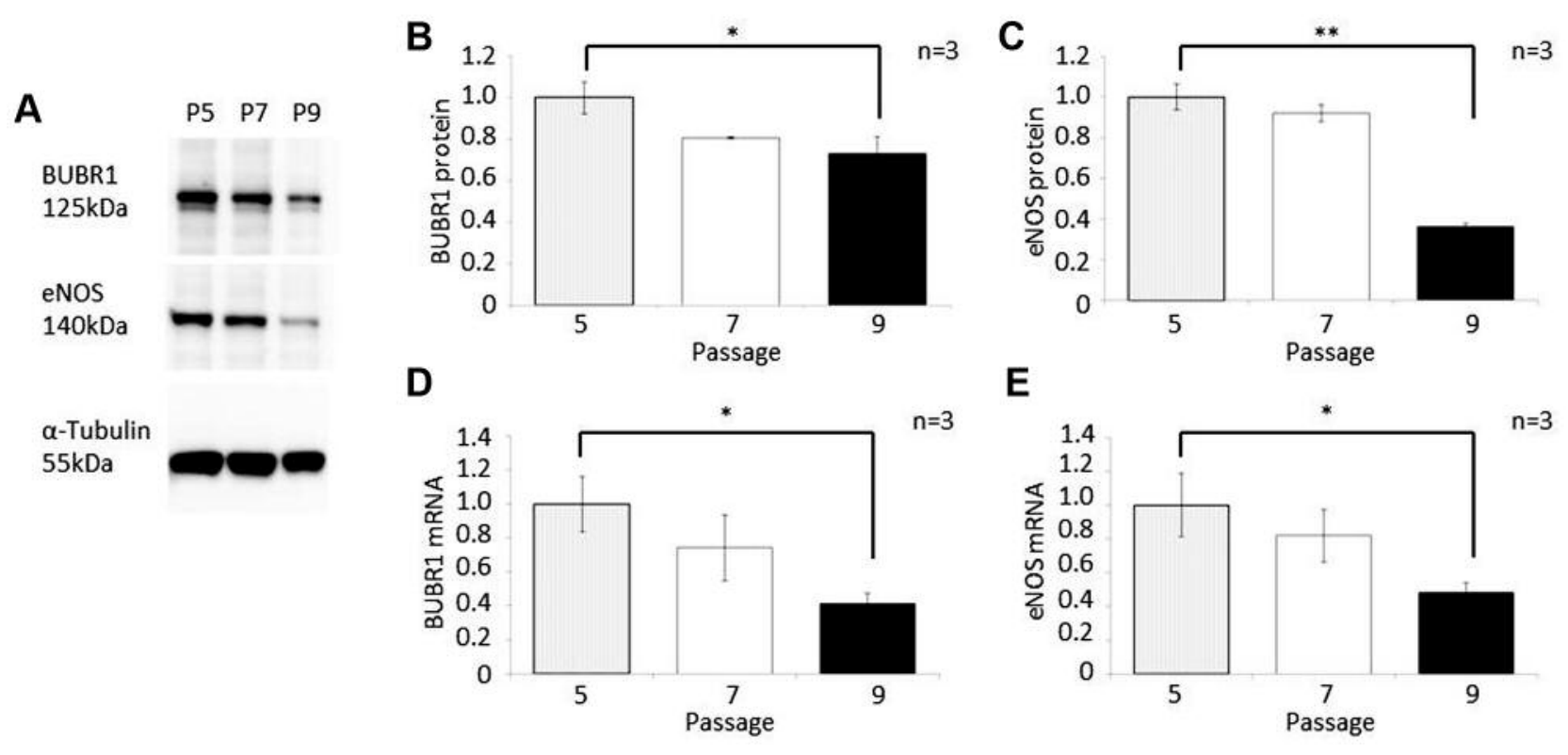

Figure 1. Budding uninhibited by benzimidazole-related 1 (BUBR1) and endothelial nitric oxide synthase (eNOS) expression in different human umbilical vein endothelial cell (HUVEC) passages. A: The relative protein expression of BUBR1 and eNOS in HUVEC passages 5, 7, and 9 was detected by western blotting. Quantification of the protein expression in (A) for B: BUBR1 and C: eNOS. Data show the mean $\pm S E$, $n=3$. mRNA expression of D: BUBRI and E: eNOS as measured by real-time polymerase chain reaction. Data are the mean $\pm S E$, $n=3$. Significantly different at $* p<0.05$ and $* * p<0.01$.

Immunohistochemical staining of BUBRI and eNOS. Formalinfixed, paraffin-embedded tissue specimens were used for immunohistochemical staining. A paraffin block that contained both cancerous tissue, which had invaded the deepest area of the stomach wall, and adjacent noncancerous tissue, was used in each case. Immunohistochemical staining was performed as previously described $(12,27)$ and used mouse monoclonal antibodies against BUBR1 (1:100; BD Biosciences) or eNOS (1:100 BD Biosciences). The intensity of cytoplasmic staining of BUBR1 was scored on a three-point scale in comparison with the staining of lymph follicles that were equally stained in all 106 specimens. Staining that was weaker than or similar to follicular staining was scored as 0 or 1 and defined as 'low' expression, while stronger staining was scored as 2 and defined as 'high' expression. The assessment of eNOS for all samples was performed as previously described (27).

Statistical analysis. All data are reported as means \pm SE. An unpaired Student's $t$-test was used to detect significant differences when two groups were compared. Statistical significance was accepted at a level of $p<0.05$.

\section{Results}

Senescence reduced the expression of both BUBR1 and eNOS in HUVECs. Western blotting was performed to determine the changes in BUBR1 and eNOS protein expression across HUVEC passages. The results show that the expression levels of both proteins were significantly reduced as HUVECs were cultured from passage 5 to 9 (Figure 1A-C. BUBR1: $p<0.05$, eNOS: $p<0.01$ ).

Next, we performed quantitative RT-PCR on sequential HUVEC passages. As the cells aged from passage 5 to 9 , the mRNA expression levels of both BUBRI and eNOS in HUVECs decreased, following the same trend as their protein expression levels (Figure 1D and E).

BUBR1 repression by siRNA transfection in HUVECs reduced eNOS expression without affecting eNOS activity. When the expression of BUBR1 was inhibited by siRNA transfection in HUVECs, the eNOS expression level was significantly reduced (passage $4, n=5$ ) (Figure 2A-C). In contrast, the expression of p-eNOS by HUVECs transfected with $B U B R 1$ siRNA was not significantly different from that of normal HUVECs (Figure 2D).

eNOS expression was reduced in the thymuses of lowBUBR1-expressing mice. Western blots were performed to measure eNOS protein expression in the thymuses of $B u b r 1^{+/+}$and Bubrl ${ }^{\mathrm{L} /-}$ mice. In Bubrl ${ }^{\mathrm{L} /-}$ mice, the eNOS expression was significantly reduced compared with Bubr ${ }^{+/+}$mice (Figure 3).

Aged Bubrl ${ }^{L /-}$ mice produce less $c G M P$ than aged WT mice. To determine the NO production ability of mice, the cGMP 


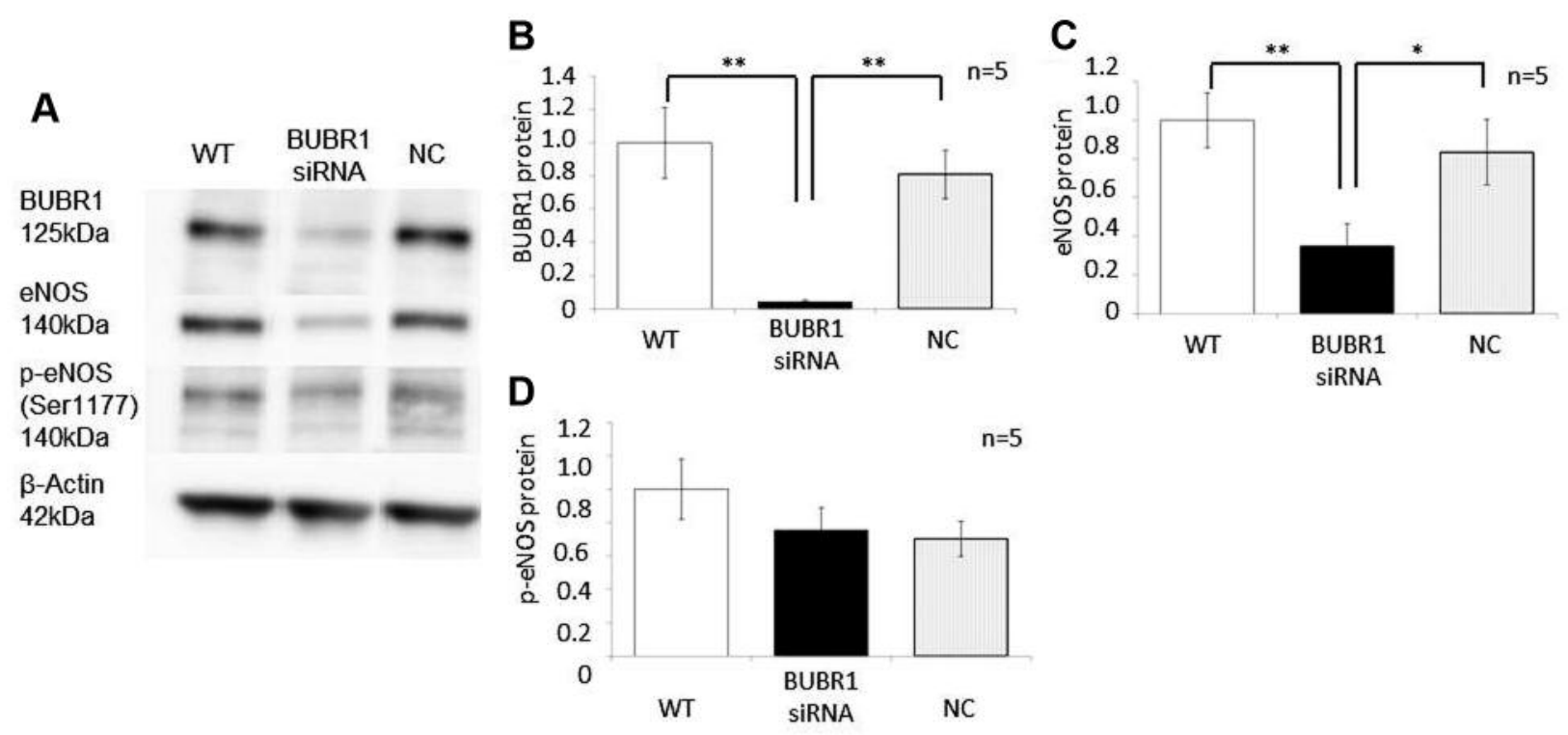

Figure 2. Effect of budding uninhibited by benzimidazole-related 1 (BUBR1) knockdown on endothelial nitric oxide synthase (eNOS) in different human umbilical vein endothelial cell (HUVEC). Passage 4 HUVECs $(n=5)$ were treated with BUBR1 siRNA, scrambled control siRNA (negative control), or left untreated (wild-type, WT). A: BUBR1 and eNOS protein expression was measured by western blotting. Quantification of the protein expression shown in (A) for B: BUBR1, C: eNOS, and D: phospho(p)-eNOS (Ser 1177). Data are the mean $\pm S E$. Significantly different at *p<0.05 and $* * p<0.01$.

A

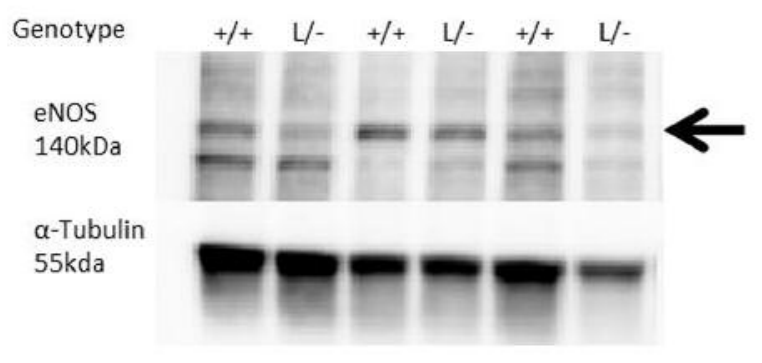

B

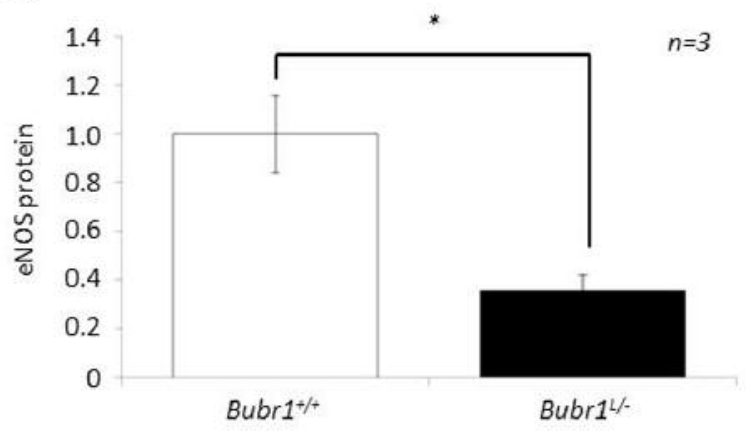

Figure 3. Endothelial nitric oxide synthase (eNOS) expression in wild-type and budding uninhibited by benzimidazole-related 1 (BUBR1)-insufficient mice. A: eNOS protein expression was measured in the thymuses of 7-week-old wild-type mice $\left(B u b r 1^{+++}\right)$and mice with low BUBR1 expression $\left(\right.$ Bubr $\left.1^{L /-}\right)$ by western blotting. B: Quantification of the eNOS protein expression shown in (A). Data are the mean \pm SE. *Significantly different at $p<0.05$.

quantity in mouse thymuses was measured by performing cGMP EIA assays. In aged mice (18-20 weeks old), the cGMP production in $B u b r 1^{\mathrm{L} /-}$ mice was significantly lower than that of $B u b r I^{+/+}$mice $(\mathrm{n}=3, p<0.05)$ (Figure 4).

VEGF-induced acceleration of eNOS phosphorylation was not significantly altered by BUBRI knockdown. HUVECs with normal or reduced (siRNA-transfected) BUBR1 levels were treated with VEGF to stimulate the expression of p-eNOS. The ratio of p-eNOS expression following VEGF treatment was not significantly different between control HUVECs and BUBRl knockdown HUVECs (Figure 5).

Human gastric cancer tissues with low BUBRI expression lacked eNOS expression. Among the 106 human gastric cancer tissues examined via immunohistochemical staining 


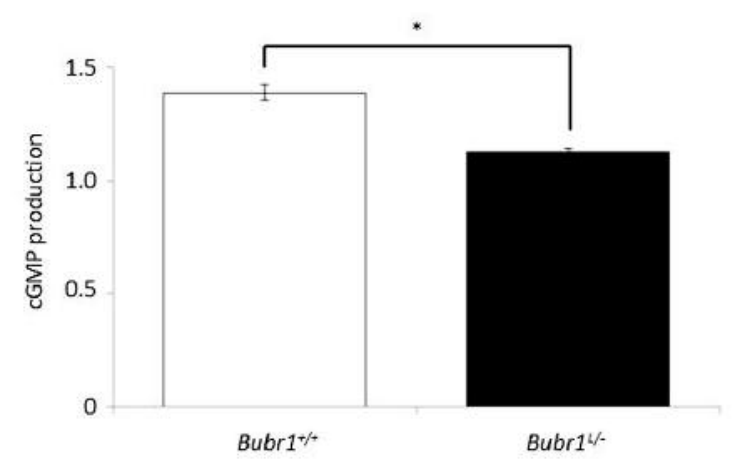

Figure 4. Guanosine 3',5' cyclic monophosphate (cGMP) production in aged (18-20 weeks old) wild-type and budding uninhibited by benzimidazole-related 1 (BUBR1)-insufficient mice. Data are the mean $\pm S E$. *Significantly different at $p<0.05$.

in this study, 98 samples had high levels of BUBR1 expression and eight had low levels of BUBR1 expression. In the eight samples with low BubR1 expression, eNOS expression was not observed at all (Figure 6A), despite the detection of both BUBR1 and eNOS expression in positive control samples (Figure 6B).

\section{Discussion}

Previous studies reported that BUBR1 has a high homology with SSK1, a cDNA fragment of which was overexpressed following laminar shear stress in HUVECs (23). Here, we showed that BUBR1 insufficiency reduced eNOS expression both in vitro and in vivo, and we also found a lack of eNOS expression in gastric cancer tissues with low BUBR1 expression.

To the best of our knowledge, there have been no previous reports on the relationship between BUBR1 and eNOS. Our novel findings in this study are summarized as follows: i) A reduction of $B U B R I$ gene expression in HUVECs reduced eNOS expression; ii) Levels of both eNOS protein and cGMP in the thymus were significantly lower in aged $B u b r l^{\mathrm{L} /-}$ mice compared with aged WT mice; iii) VEGF-induced phosphorylation of eNOS was not influenced by BUBR1 inhibition; and iv) eNOS expression was not detected in BUBR1-negative cells within human gastric cancer tissues.

In HUVECs, steady laminar shear stress for $6 \mathrm{~h}$ induces an up-regulation of the SSK1 gene, which encodes for a kinase protein related to BUBR1 (23). Shear stress also increases eNOS transcription via a pathway involving activation of the tyrosine kinase c-SRC and extracellular signal-related kinase 1/2 (ERK1/2). After several hours of stimulation by shear stress, the expression levels of both eNOS mRNA and protein were increased (28).
Here, we first evaluated the expression of BUBR1 and eNOS in HUVECs to investigate the effect of aging. Aged HUVECs exhibited significantly lower mRNA and protein levels of both eNOS and BUBR1 than young HUVECs (Figure 1). Additionally, BUBRI siRNA-transfected endothelial cells showed a significantly lower eNOS expression compared with control HUVECs, suggesting that the down-regulation of eNOS observed in aged HUVECs may be caused by an age-dependent reduction in BUBR1 level (Figure 2A-C).

Next, we investigated the effect of BUBR1 insufficiency on eNOS expression in vivo. We previously generated the Bubr $1^{\mathrm{L} /-}$ mouse strain in which BUBR1 expression in the testis was reduced to $16.8 \%$ of the normal level (14). BUBR 1 and eNOS were reported to be expressed abundantly in the murine thymus (29), therefore we performed western blots to assess the eNOS expression in the thymus of Bubrl ${ }^{\mathrm{L} /-}$ mice. The results revealed that BUBR1 insufficiency in vivo also reduced eNOS expression.

Biochemical analysis of $B u b r l^{\mathrm{L} /-}$ mouse thymuses demonstrated significantly reduced the level of cGMP, a second messenger for NO. Because eNOS activity is regulated via phosphorylation and previous work showed that p-eNOS is reduced in aged HUVECs (21), we examined the effect of $B U B R 1$ siRNA on eNOS phosphorylated at Ser1177. Despite the lower level of total eNOS in BUBRI siRNA-transfected HUVECs compared with control HUVECs, we found no significant difference between their p-eNOS expressions. Together, these findings suggest that the observed agedependent decrease of p-eNOS in HUVECs is not mediated through an age-dependent decrease in BUBR1.

The VEGF-phosphoinositide 3-kinase/protein kinase B pathway has been implicated in regulating eNOS enzyme activity through phosphorylation (17). Based on this connection, we examined the effect of BUBR1 insufficiency on VEGF-stimulated eNOS phosphorylation in vitro. The results indicate that BUBR1 insufficiency did not affect the level of VEGF-stimulated eNOS phosphorylation. These findings suggest that another pathway likely exists between BUBR1 and eNOS. Abnormal BUBR1 expression may be an important factor for DNA aneuploidy and may contribute to chromosomal instability (19-23). Additionally, abnormal BUBR1 expression was found in gastric cancer. Previous work on gastric cancer tissues found conflicting results concerning the relative level of eNOS expression in tumour tissue compared with that in normal tissue; one study reported that eNOS expression was lower in tumour tissue (30), while another found that it was higher (31). Here, we examined the relationship between BUBR1 expression and eNOS expression in gastric cancer tissues. The immunohistochemical staining results revealed that there was no eNOS expression in the gastric cancer tissues with low BUBR1 expression, which may support our in vitro and in vivo data. 

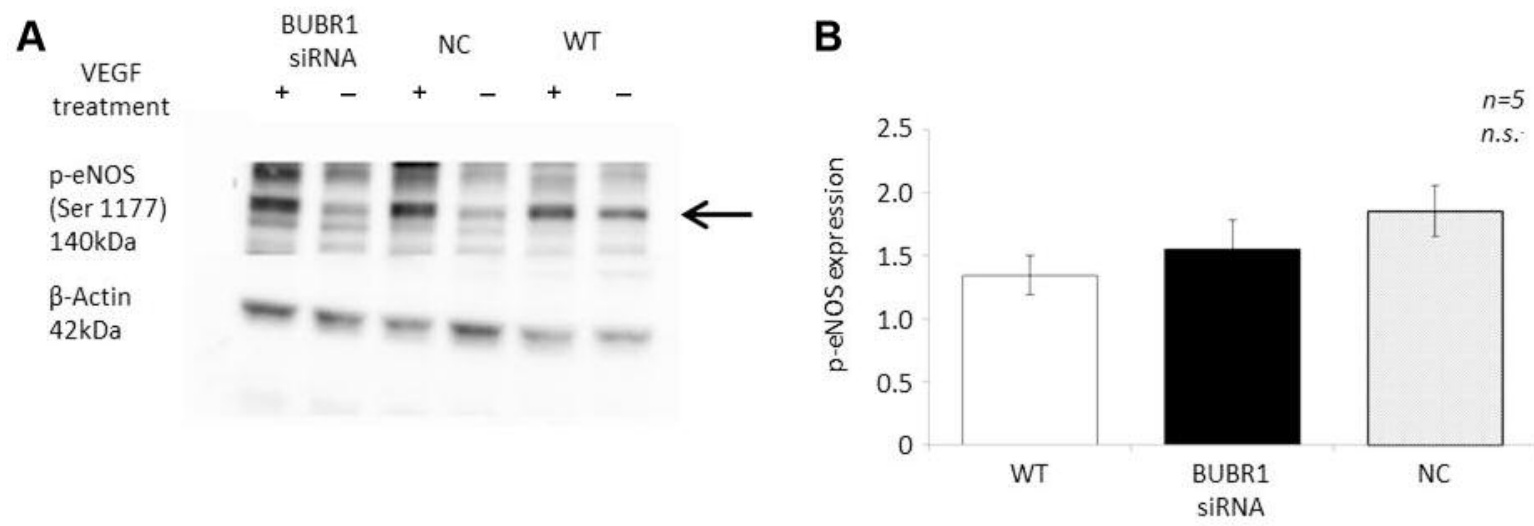

Figure 5. Vascular endothelial growth factor (VEGF)-stimulated endothelial nitric oxide synthase (eNOS) activity in budding uninhibited by benzimidazole-related 1 (BUBR1)-knockdown and wild-type (WT) human umbilical vein endothelial cells (HUVECs). Passage 3 HUVECs ( $n=5$ per group) that had been transfected with BUBR1 siRNA, scrambled control siRNA (negative control, NC), or left untransfected (WT) were treated with $V E G F$ or left untreated. A: The expression of phospho-eNOS (Ser 1177; p-eNOS) was measured by western blotting. B: Quantification of the peNOS expression shown in (A). Data show the mean $\pm S E(B), n=3$. No significant differences were found.

A
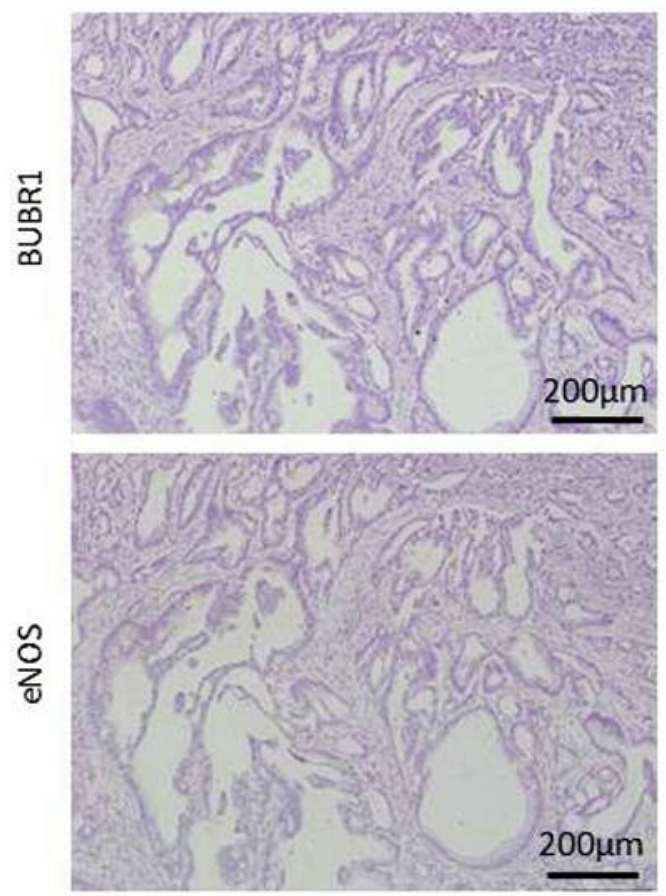

B
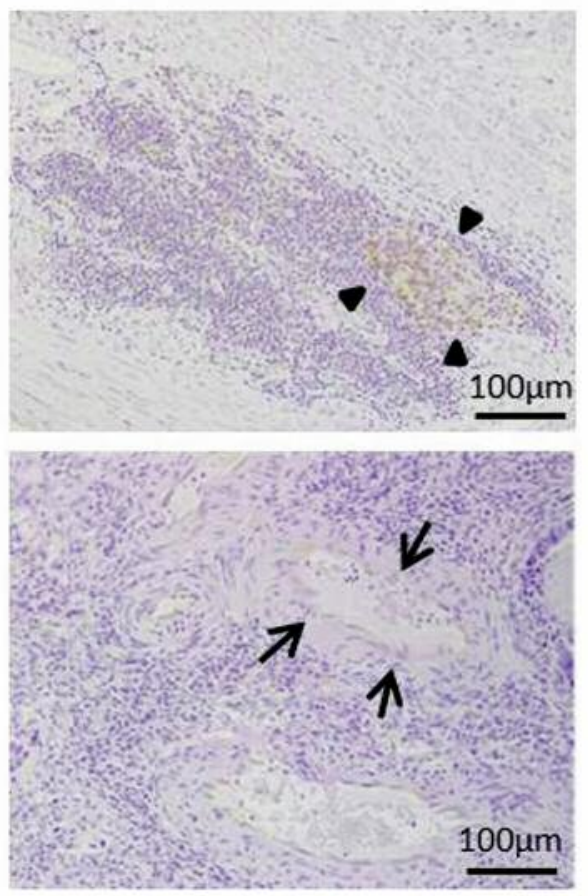

Figure 6. Budding uninhibited by benzimidazole-related 1 (BUBR1) and endothelial nitric oxide synthase (eNOS) expression in tissues of gastric cancer from patients. The expression of BUBR1 and eNOS in gastric cancer tissues was investigated by immunohistochemistry. In 106 tissue samples, 98 showed high BUBR1 expression and eight showed low BUBR1 expression. A: There was no eNOS expression in the tissues with low-BUBR1 expression. B: Positive control images. The BUBR1-positive control section shows a lymphoid follicle, and the eNOS-positive control shows lymph duct epithelium.

The pathway by which BUBR1 reduction is connected to eNOS repression remains unclear. The molecular mechanisms responsible for the down-regulation of NO production and its biological activity in BUBR1 insufficiency are also unknown and remain to be studied. This study is a first step into analysing the function of BUBR1 in aging and angiogenesis in view of its relationship with eNOS. 


\section{Sources of Funding}

This work was supported in part by a Grant-in-Aid for Scientific Research (C) from the Japan Society for the Promotion of Science (Grant No. 25462164 to TM) and Uehara Memorial Foundation.

\section{Acknowledgements}

The Authors thank Natsumi Maeda for assistance with the animal experiments and thank Eriko Iwata for technical assistance. We also thank Katie Oakley, Ph. D., from Edanz Group (www.edanzediting.com/ac) for editing a draft of this manuscript.

\section{References}

1 de Magalhães JP: How ageing processes influence cancer. Nat Rev Cancer 13: 357-365, 2013.

2 Aunan JR, Cho WC and Søreide K: The biology of aging and cancer: A brief overview of shared and divergent molecular hallmarks. Aging Dis 8: 628-642, 2017.

3 López-Otín C, Blasco MA, Partridge L, Serrano M and Kroemer G: The hallmarks of aging. Cell 153: 1194-1217, 2013.

4 Blackwell KA, Sorenson JP, Richardson DM, Smith LA, Suda $\mathrm{O}$, Nath K and Katusic ZS: Mechanisms of aging-induced impairment of endothelium-dependent relaxation: Role of tetrahydrobiopterin. Am J Physiol Heart Circ Physiol 287: H2448-2453, 2004

5 Matsumoto T, Baker DJ, d'Uscio LV, Mozammel G, Katusic ZS and van Deursen JM: Aging-associated vascular phenotype in mutant mice with low levels of BUBR1. Stroke 38: 1050-1056, 2007.

6 Folkman J: Tumor angiogenesis: therapeutic implications. N Engl J Med 285: 1182-1186, 1971.

7 Yonemitsu Y, Matsumoto T, Itoh H, Okazaki J, Uchiyama M, Yoshida K, Onimaru M, Onohara T, Inoguchi H, Kyuragi R, Shimokawa M, Ban H, Tanaka M, Inoue M, Shu T, Hasegawa M, Nakanishi Y and Maehara Y: DVC1-0101 to treat peripheral arterial disease: A phase I/IIa open-label dose-escalation clinical trial. Mol Ther 21: 707-714, 2013.

8 Morishita R, Makino H, Aoki M, Hashiya N, Yamasaki K, Azuma J, Taniyama Y, Sawa Y, Kaneda Y and Ogihara T: A phase I/IIa clinical trial of therapeutic angiogenesis using hepatocyte growth factor gene transfer to treat critical limb ischemia. Arterioscler Thromb Vasc Biol 31: 713-720, 2011.

9 Baumgartner I, Pieczek A, Manor O, Blair R, Kearney M, Walsh $\mathrm{K}$ and Isner JM: Constitutive expression of phVEGF165 after intramuscular gene transfer promotes collateral vessel development in patients with critical limb ischemia. Circulation 97: 1114-1123, 1998.

10 Shoji T, Yonemitsu Y, Komori K, Tanii M, Itoh H, Sata S, Shimokawa H, Hasegawa M, Sueishi $\mathrm{K}$ and Maehara Y: Intramuscular gene transfer of FGF-2 attenuates endothelial dysfunction and inhibits intimal hyperplasia of vein grafts in poor-runoff limbs of rabbit. Am J Physiol Heart Circ Physiol 285: H173-182, 2003.

$11 \mathrm{Yu} \mathrm{H}$ : Regulation of APC-CDC20 by the spindle checkpoint Curr Opin Cell Biol 14: 706-714, 2002.

12 Ando K, Kakeji Y, Kitao H, Iimori M, Zhao Y, Yoshida R, Oki E, Yoshinaga K, Matsumoto T, Morita M, Sakaguchi Y and
Maehara Y: High expression of BUBR1 is one of the factors for inducing DNA aneuploidy and progression in gastric cancer. Cancer Sci 101: 639-645, 2010.

13 Baker DJ, Jeganathan KB, Cameron JD, Thompson M, Juneja S, Kopecka A, Kumar R, Jenkins RB, de Groen PC, Roche P and van Deursen JM: BUBR1 insufficiency causes early onset of aging-associated phenotypes and infertility in mice. Nat Genet 36: 744-749, 2004.

14 Okadome J, Matsumoto T, Yoshiya K, Matsuda D, Tamada K, Onimaru M, Nakano K, Egashira K, Yonemitsu Y and Maehara Y: BUBR1 insufficiency impairs angiogenesis in aging and in experimental critical limb ischemic mice. J Vasc Surg 68: 576586.e1, 2018

15 Ignarro LJ: Physiological significance of endogenous nitric oxide. Semin Perinatol 15: 20-26, 1991.

16 Förstermann U and Sessa WC: Nitric oxide synthases: Regulation and function. Eur Heart J 33: 829-837, 837a-837d, 2012.

17 Fulton D, Gratton JP, McCabe TJ, Fontana J, Fujio Y, Walsh K, Franke TF, Papapetropoulos A and Sessa WC: Regulation of endothelium-derived nitric oxide production by the protein kinase AKT. Nature 399: 597-601, 1999.

18 Atochin DN and Huang PL: Endothelial nitric oxide synthase transgenic models of endothelial dysfunction. Pflugers Arch 460: 965-974, 2010

19 Kashiwagi S, Izumi Y, Gohongi T, Demou ZN, Xu L, Huang PL, Buerk DG, Munn LL, Jain RK and Fukumura D: NO mediates mural cell recruitment and vessel morphogenesis in murine melanomas and tissue-engineered blood vessels. J Clin Invest 115: 1816-1827, 2005.

20 Selleri C, Maciejewski JP, Montuori N, Ricci P, Visconte V, Serio B, Luciano L and Rotoli B: Involvement of nitric oxide in farnesyltransferase inhibitor-mediated apoptosis in chronic myeloid leukemia cells. Blood 102: 1490-1498, 2003.

21 Hoffmann J, Haendeler J, Aicher A, Rössig L, Vasa M, Zeiher $\mathrm{AM}$ and Dimmeler S: Aging enhances the sensitivity of endothelial cells toward apoptotic stimuli: Important role of nitric oxide. Circ Res 89: 709-715, 2001.

22 Tschudi MR, Barton M, Bersinger NA, Moreau P, Cosentino F, Noll G, Malinski T and Lüscher TF: Effect of age on kinetics of nitric oxide release in rat aorta and pulmonary artery. J Clin Invest 98: 899-905, 1996.

23 Donadelli R, Benatti L, Remuzzi A, Morigi M, Gullans SR, Benigni A, Remuzzi $G$ and Noris M: Identification of a novel gene-SSK1-in human endothelial cells exposed to shear stress. Biochem Biophys Res Commun 246: 881-887, 1998.

24 Raveendran M, Wang J, Senthil D, Wang J, Utama B, Shen Y, Dudley D, Zhang Y and Wang XL: Endogenous nitric oxide activation protects against cigarette smoking induced apoptosis in endothelial cells. FEBS Lett 579: 733-740, 2005.

25 Baker DJ, Dawlaty MM, Wijshake T, Jeganathan KB, Malureanu L and van Ree JH, Crespo-Diaz R, Reyes S, Seaburg L, Shapiro V, Behfar A, Terzic A, van de Sluis B and van Deursen JM: Increased expression of BubR1 protects against aneuploidy and cancer and extends healthy lifespan. Nat Cell Biol 15: 96-102, 2013.

26 Cudmore MJ1, Hewett PW, Ahmad S, Wang KQ, Cai M, Al-Ani B, Fujisawa T, Ma B, Sissaoui S, Ramma W, Miller MR, Newby DE, Gu Y, Barleon B, Weich $\mathrm{H}$ and Ahmed A: The role of heterodimerization between VEGFR-1 and VEGFR-2 in the regulation of endothelial cell homeostasis. Nat Commun 3: 972, 2012. 
27 Wang YZ, Cao YQ, Wu JN, Chen M and Cha XY: Expression of nitric oxide synthase in human gastric carcinoma and its relation to p53, PCNA. World J Gastroenterol 11: 46-50, 2005.

28 Davis ME, Grumbach IM, Fukai T, Cutchins A and Harrison DG: Shear stress regulates endothelial nitric-oxide synthase promoter activity through nuclear factor kappaB binding. J Biol Chem 279: 163-168, 2004.

29 Li W, Lan Z, Wu H, Wu S, Meadows J, Chen J, Zhu V and Dai $\mathrm{W}$ : BUBR1 phosphorylation is regulated during mitotic checkpoint activation. Cell Growth Differ 10: 769-775, 1999.

30 Doi C, Noguchi Y, Marat D, Saito A, Fukuzawa K, Yoshikawa $\mathrm{T}$, Tsuburaya A and Ito T: Expression of nitric oxide synthase in gastric cancer. Cancer Lett 144: 161-167, 1999.
31 Wang L, Shi GG, Yao JC, Gong W, Wei D, Wu T-T, Ajani JA, Huang $\mathrm{S}$ and Xie K: Expression of endothelial nitric oxide synthase correlates with the angiogenic phenotype of and predicts poor prognosis in human gastric cancer. Gastric Cancer 8: 18-28, 2005.
Received September 18, 2018

Revised October 11, 2018

Accepted October 15, 2018 